\title{
All-suture anchors versus metal suture anchors in the arthroscopic treatment of traumatic anterior shoulder instability: A comparison of mid-term outcomes
}

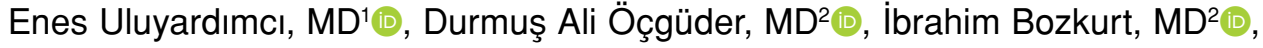 \\ Selçuk Korkmazer, $\mathrm{MD}^{3}$ iD, Mahmut Uğurlu, $\mathrm{MD}^{2}$ (1) \\ ${ }^{1}$ Department of Orthopedics and Traumatology, Develi Hatice-Muammer Kocatürk State Hospital, Kayseri, Turkey \\ ${ }^{2}$ Department of Orthopedics and Traumatology, Ankara City Hospital, Ankara, Turkey \\ ${ }^{3}$ Department of Orthopedics and Traumatology, Ankara Dr. Sami Ulus Training and Research Hospital, Ankara, Turkey
}

Arthroscopic treatment of anterior shoulder instability has become the standard surgical technique in the treatment of traumatic anterior shoulder instability, as the clinical outcomes are similar or superior to those of open repair. ${ }^{[1,2]}$ Several fixation materials have been used to facilitate the arthroscopic technique in this process. Arthroscopic capsulorraphies performed with $\mathrm{U}$ nails and various transglenoid suture techniques have resulted in high complication rates. ${ }^{[3]}$ This situation has prompted the orthopedic industry to investigate different fixation materials and the suture anchor technique has become the gold standard treatment for capsulolabral lesions in recent years. ${ }^{[4]}$

Received: March 24, 2020

Accepted: June 18, 2020

Published online: January 06, 2021

Correspondence: Enes Uluyardımcı, MD. Bahçelievler Mah., Bahçelievler Cad., Çavdaroğlu Apt, No: 21, 38280 Talas, Kayseri, Türkiye.

E-mail: enesuluyardmc7@hotmail.com

Doi: 10.5606/ehc.2021.75027

Citation: Uluyardımcı E, Öçgüder DA, Bozkurt I, Korkmazer S, Uğurlu $M$. All-suture anchors versus metal suture anchors in the arthroscopic treatment of traumatic anterior shoulder instability: a comparison of mid-term outcomes. Jt Dis Relat Surg 2021;32(1):101-107.

(O2021 All right reserved by the Turkish Joint Diseases Foundation

This is an open access article under the terms of the Creative Commons Attribution-NonCommercial License, which permits use, distribution and reproduction in any medium, provided the original work is properly cited and is not used for commercial purposes (http://creativecommons.org/licenses/by-nc/4.0/).

\section{ABSTRACT}

Objectives: This study aims to compare metal suture anchors and all-suture anchors clinically and radiologically in arthroscopic Bankart repair.

Patients and methods: In this retrospective study, 67 patients (61 males, 6 females; mean age 26.0 \pm 5.8 ; range, 18 to 43 years) who underwent arthroscopic Bankart repair between April 2009 and October 2016 were divided into two groups depending on the type of the suture anchor used in different periods. Group A comprised 32 patients with arthroscopic Bankart repair performed with metal suture anchors, and Group B comprised 35 patients with arthroscopic Bankart repair performed with all-suture anchors. The patients were clinically evaluated using Rowe scores, Constant scores, redislocation rates, and positive apprehension test rates. Radiographic evaluation was performed using the Samilson-Prieto classification to observe the development of glenohumeral osteoarthritis.

Results: The mean follow-up period was $41.1 \pm 10.4$ (range, 30 to 60 ) months in Group A, and 39.6 \pm 9.4 (range, 28 to 60 ) months in Group B, with no significant difference between the two groups $(p=0.559)$. No significant difference was observed between Group A and Group B in terms of mean Rowe score $(89.2 \pm 13.8$ [range, 40 to 100 ] vs. $88.7 \pm 16.9$ [range, 25 to 100 ]; $\mathrm{p}=0.895$ ) or Constant score $(87.2 \pm 8.9$ [range, 48 to 96 ] vs. $86.9 \pm 9.0$ [range, 46 to 96]; $\mathrm{p}=0.878$ ), which were the clinical outcomes at the final follow-up examination. Postoperative redislocation rates $(3.1 \%$ vs. $2.9 \%, \mathrm{p}=1.0)$ and positive apprehension test rates $(6.3 \%$ vs. $8.6 \%, \mathrm{p}=1.0$ ) were found to be similar in both groups. According to the Samilson-Prieto classification, there was no evidence of glenohumeral osteoarthritis in any of the patients in either group.

Conclusion: Satisfactory outcomes were obtained with the use of all-suture anchors in arthroscopic Bankart repair for traumatic anterior shoulder instability. All-suture anchors and metal suture anchors, have similar outcomes in the mid-term and all-suture anchors are a reliable and effective option for arthroscopic Bankart repair.

Keywords: All-suture anchor, Bankart lesion, metallic anchor, shoulder instability. 
At this point, the availability of a wide variety of suture anchors such as metal, biodegradable, and all-suture anchors has resulted in much research on this subject. All-suture anchors without a rigid component have come into more widespread use in recent years due to features such as protecting the bone stock, facilitating better postoperative imaging, and allowing revision surgery when necessary. ${ }^{[5]}$ Although all-suture anchors have the disadvantages of potential cyst formation and tunnel expansion, satisfactory short-term clinical results have been published..$^{[6,7]}$ Willemot et al. ${ }^{[5]}$ used all-suture anchors for labral lesions in the arthroscopic treatment of 20 patients and reported successful clinical and radiological outcomes after a mean follow-up of 19 (range, 12 to 28 ) months. Lee et al. ${ }^{[7]}$ compared all-suture anchors and conventional biodegradable suture anchors in arthroscopic Bankart repair and reported clinically similar outcomes in both groups after a two-year follow-up.

To the best of our knowledge, there are no studies in the literature in which metal suture anchors and the relatively new all-suture anchors have been clinically and radiologically compared in terms of the arthroscopic treatment of traumatic anterior shoulder instability. Therefore, in this study, we aimed to compare metal suture anchors and all-suture anchors clinically and radiologically in arthroscopic Bankart repair.

\section{PATIENTS AND METHODS}

Patients who underwent arthroscopic Bankart repair due to traumatic anterior shoulder instability were recruited in Ankara Atatürk Training and Research Hospital, Department of Orthopedics and Traumatology between April 2009 and October 2016 for this retrospective study. All operations were performed by a single surgeon. This surgeon has used all-suture anchors in all arthroscopic Bankart repair procedures since late 2013 when all-suture anchors became available in our country. Before that time, in the period between 2009 and 2013, metal suture anchors were used in all patients. The study inclusion criteria were determined as patients with (i) an isolated Bankart lesion without any other labral injuries identified in arthroscopic examination; (ii) a minimum follow-up period of at least two years; (iii) and a maximum follow-up period of five years. All patients had recurrent anterior shoulder dislocation and were active individuals suffering from restrictions in daily activities because of shoulder instability. The study exclusion criteria were determined as patients (i) aged $<18$ years; (ii) with preoperative glenohumeral osteoarthritis; (iii) requiring a bone augmentation procedure due to $>25 \%$ glenoid bone loss; ${ }^{\left[{ }^{[8]}\right.}$ (iv) requiring a remplissage procedure due to an engaged Hill-Sachs lesion; ${ }^{[8]}$ (v) with a concomitant superior labral anterior posterior (SLAP) tear lesion requiring repair, or (vi) with generalized joint laxity or multidirectional instability. ${ }^{[9]}$ Group A comprised patients applied with arthroscopic Bankart repair with metal suture anchors (3.5 mm; TWINFIX ${ }^{\mathrm{TM}}$, Smith\&Nephew Inc., Andover, MA, USA) between 2009 and 2013. Group B comprised patients applied with arthroscopic Bankart repair with all-suture anchors $(2.9 \mathrm{~mm}$; JuggerKnot ${ }^{\circledR}$, Biomet Inc., Warsaw, IN, USA) between 2013 and 2016. Of 66 patients operated on with metal suture anchors, a total of 34 were excluded; 12 patients with a follow-up period of less than two years or lost to follow-up, two aged $<18$ years, three with SLAP tear repair, three with Latarjet procedure, two with remplissage procedure, five with generalized joint laxity or multidirectional instability, and seven with a follow-up period of more than five years. Of 71 patients operated with all-suture anchors, a total of 36 were excluded; seven patients with a follow-up period of less than two years or lost to follow-up, four aged $<18$ years, six with SLAP tear repair, three with Latarjet procedure, eight with remplissage procedure, seven with generalized joint laxity or multidirectional instability, and one patient with a follow-up period of more than five years. Finally, a total of 67 patients (61 males, 6 females; mean age $26.0 \pm 5.8$; range, 18 to 43 years) were included as 32 patients in Group A and 35 patients in Group B. The study protocol was approved by the Ankara Yildırım Beyazıt University Faculty of Medicine Ethics Committee Ethics Committee (Date: 23.11.2016, No: 26379996/256). A written informed consent was obtained from each patient. The study was conducted in accordance with the principles of the Declaration of Helsinki.

Surgical procedures were performed under general anesthesia with the patient in the beach chair position. First, a posterior portal was opened and intra-articular imaging was performed. The second portal was opened antero-superiorly, and diagnostic arthroscopy was performed. Then, the third portal was opened $3 \mathrm{~cm}$ inferior of the antero-superior portal. The retracted labroligamentous complex was mobilized and elevated to the level of approximately six o'clock using a Bankart-Chisel and a radiofrequency device. When placing the suture anchors in the anterior glenoid rim, for the right shoulder, the first anchor was placed to be in the 5:30 position and the second anchor 
in the 4:00 position. Care was taken that the anchors were placed towards the cartilage surface at approximately $2 \mathrm{~mm}$. Fixation was performed with sliding knots which would slide proximal to the capsule-labral complex. Depending on the size of the labral lesion, when necessary, a third anchor was placed in the 2:30 position. While metal suture anchors were placed by direct nailing, all-suture anchors were placed after being drilled with a drill specific to the size of the anchor (Figure 1). The distinctive feature of all-suture anchors is that they consist entirely of suture. It is drawn back to some extent after the initial placement to the glenoid and the suture material is fixed by being squeezed in the bone tunnel formed (Figure 2). In Group B, pullout occurred in one anchor each of two patients when applying the pulling force for fixation of the anchor in the bone tunnel after placement. Repair was applied in these two patients again using all-suture anchors with the creation of new tunnels.

Postoperative immobilization was ensured for all patients with application of a Velpeau bandage, allowing hand, wrist, and elbow movements during that period. Between the third and sixth weeks, the patients received strengthening training of pendulum movements, submaximal isometric exercises, and passive and active-assisted range of motion exercises. The patients were allowed free movement of the shoulder after the sixth week except for advanced external rotation and extension

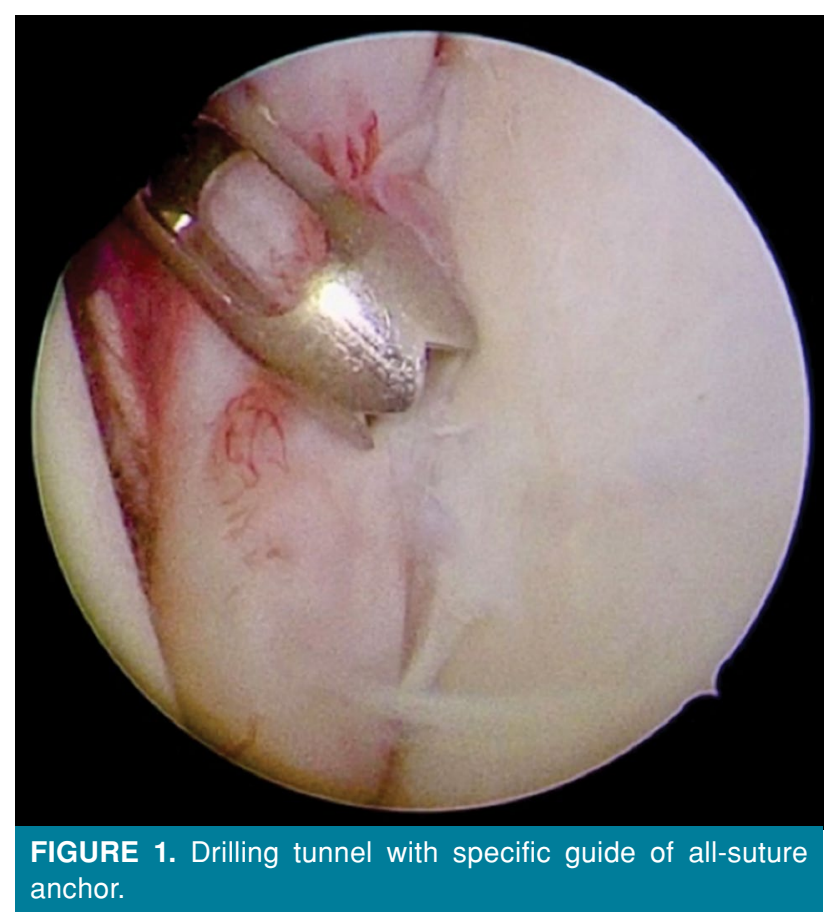

movements. Plyometric exercises and swimming were permitted in the fourth month. The patients were asked not to return to sports until after the sixth month.

Information regarding the patient age, sex, affected side, dominant hand, age at first dislocation, and the time from first dislocation to surgery were noted. All patients were clinically evaluated with the Rowe et al., ${ }^{[10]}$ and Constant and Murley ${ }^{[11]}$ scores at the postoperative final follow-up examination. The Rowe score consists of three sections: stability, motion, and function. A score of 90-100 is considered excellent, 89-75 good, 74-51 fair, and $\leq 50$ poor. The Constant score consists of pain, activity level, arm positioning, strength of abduction, and range of motion. A score of 90-100 is considered excellent, 80-89 good, 70-79 fair, and $\leq 69$ poor. Redislocation rates and positive apprehension test rates were noted. Anteroposterior X-ray at internal and external rotation, West Point $X$-ray, and magnetic resonance imaging (MRI) were obtained routinely from all patients before the surgery. The development of glenohumeral osteoarthritis was evaluated according to the Samilson-Prieto classification, using the anteroposterior radiographs at internal rotation and external rotation obtained in the postoperative final follow-up examination. ${ }^{[12]}$ Clinical and radiological evaluations were performed by a physician assistant who was not associated with the study.

(a)

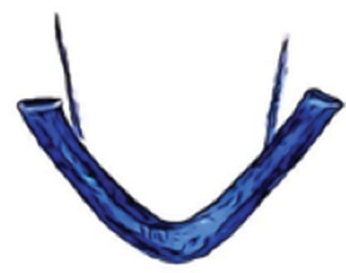

(b)

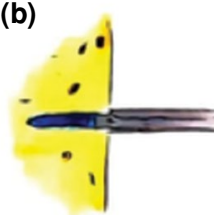




\begin{tabular}{|c|c|c|c|c|c|c|c|}
\hline \multicolumn{8}{|c|}{$\begin{array}{c}\text { TABLE I } \\
\text { Preoperative patient demographics }\end{array}$} \\
\hline & \multicolumn{3}{|c|}{ Group A $(n=32)$} & \multicolumn{3}{|c|}{ Group B $(n=35)$} & \multirow[b]{2}{*}{$p$} \\
\hline & $\mathrm{n}$ & $\%$ & Mean $\pm S D$ & $\mathrm{n}$ & $\%$ & Mean $\pm S D$ & \\
\hline Age at operation (year) & & & $26.3 \pm 5.8$ & & & $25.7 \pm 5.9$ & 0.675 \\
\hline Sex & & & & & & & 0.675 \\
\hline Male & 30 & 93.8 & & 31 & 88.6 & & \\
\hline Female & 2 & & & 4 & & & \\
\hline Affected side & & & & & & & 1 \\
\hline Right & 26 & & & 29 & & & \\
\hline Left & 6 & & & 6 & & & \\
\hline Dominant hand & 26 & & & 28 & & & 1 \\
\hline Age at first dislocation (year) & & & $22.6 \pm 4.4$ & & & $22.1 \pm 4.3$ & 0.634 \\
\hline Time from first dislocation to surgery (month) & & & $37.7 \pm 32.4$ & & & $36.8 \pm 28.2$ & 0.905 \\
\hline SD: Standard deviation. & & & & & & & \\
\hline
\end{tabular}

\section{Statistical analysis}

Statistical analysis was performed using the IBM SPSS version 23.0 software (IBM Corp., Armonk, NY, USA). Categorical measurements were summarized as numbers and percentages and continuous measurements as mean \pm standard deviation values. The chi-square test or Fisher's test was used in the comparison of categorical variables. Distributions between the groups were checked in the comparison of continuous measurements. The Student's t-test was used for variables with parametric distribution and the Mann-Whitney $U$ test for variables without parametric distribution. Correlations between the variables were determined with the Pearson correlation test. Correlation coefficient assessment was considered: a high correlation between the variables if $r \geq 0.91$; good correlation if $0.90 \leq r \geq 0.71$; medium correlation if $0.70 \leq \mathrm{r} \geq 0.51$; low correlation if $0.50 \leq r \geq 0.31$; and no correlation if $r \leq 0.3$. The level of statistical significance was taken as $p<0.05$ in all tests.

\section{RESULTS}

There was no significant difference between the two groups in terms of preoperative demographic data (Table I). The mean follow-up period was $41.1 \pm 10.4$ (range, 30 to 60 ) months in Group A and $39.6 \pm 9.4$ (range, 28 to 60 ) months in Group B with no significant difference between the two groups $(\mathrm{p}=0.559)$. The mean Rowe score was $89.2 \pm 13.8$ (range, 40 to 100) in Group A and $88.7 \pm 16.9$ (range, 25 to 100) in Group B at the final follow-up examination, with no significant difference between the two groups $(\mathrm{p}=0.895)$. The mean Constant score was $87.2 \pm 8.9$ (range, 48 to 96 ) in Group A and 86.9 \pm 9.0 (range, 46 to 96 ) in Group B and the two groups were found to be similar ( $p=0.878)$. Clinically good or excellent outcomes were obtained in $93.7 \%$ of the patients in Group A and in $94.2 \%$ of the patients in Group B. Postoperative redislocation was observed in one patient in both groups. Redislocation occurred as a result of a motor vehicle accident in the Group A

\section{TABLE II}

Comparison of postoperative clinical outcomes between patients operated with metal and all-suture anchors

\begin{tabular}{|c|c|c|c|c|c|c|c|c|c|}
\hline & \multicolumn{4}{|c|}{ Group A $(n=32)$} & \multicolumn{4}{|c|}{ Group B $(n=35)$} & \multirow[b]{2}{*}{$p$} \\
\hline & $\mathrm{n}$ & $\%$ & Mean $\pm S D$ & Mean & $\mathrm{n}$ & $\%$ & Mean \pm SD & Mean & \\
\hline Duration of follow-up (month) & & & $41.1 \pm 10.4$ & & & & $39.6 \pm 9.4$ & & 0.559 \\
\hline Number of anchors & & & & 2.6 & & & & 2.7 & 1 \\
\hline Number of redislocation & 1 & 3.1 & & & 1 & 2.9 & & & 1 \\
\hline Number of positive in apprehension test & 2 & 6.3 & & & 3 & 8.6 & & & 1 \\
\hline Rowe score & & & $89.2 \pm 13.8$ & & & & $88.7 \pm 16.9$ & & 0.895 \\
\hline Constant score & & & $87.2 \pm 8.9$ & & & & $86.9 \pm 9.0$ & & 0.878 \\
\hline
\end{tabular}




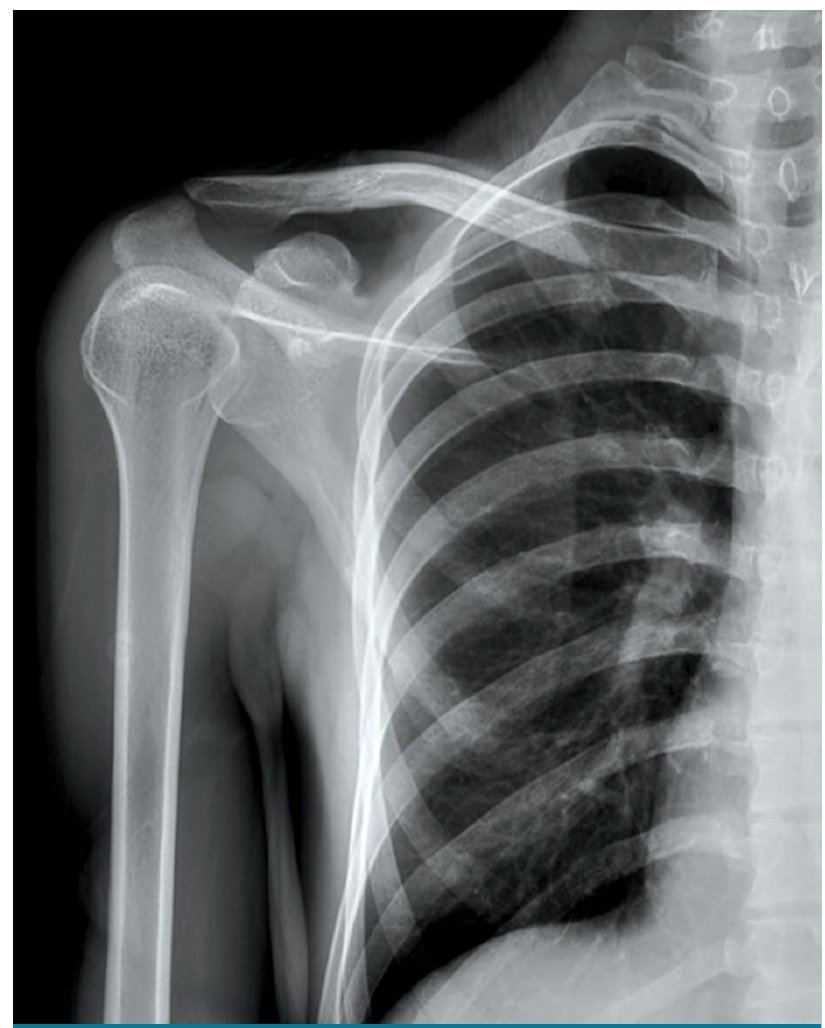

FIGURE 3. Postoperative direct radiograph of a patient treated with all-suture anchors.

patient and a direct collision when playing football in the Group B patient. Open Latarjet reconstruction was performed on both patients. Apprehension test positivity was determined in two patients in Group A and in three patients in Group B (Table II). According to the Samilson-Prieto classification, there was no evidence of glenohumeral osteoarthritis in any of the patients in either group (Figure 3). There was no statistically significant correlation between the patient age at operation and age at first dislocation and the clinical scores. A high correlation was identified between the Rowe score and the Constant score $(\mathrm{r}=0.91 ; \mathrm{p}=0.0001)$.

\section{DISCUSSION}

The results of this study demonstrated that metal suture anchors and all-suture anchors had comparable clinical outcomes in arthroscopic Bankart repair at the end of mean follow-up periods of $41.1 \pm 10.4$ (range, 30 to 60 ) months and 39.6 \pm 9.4 (range, 28 to 60) months, respectively. Satisfactory outcomes were obtained with the use of all-suture anchors in arthroscopic Bankart repair for traumatic anterior shoulder instability, and all-suture anchors are a reliable and effective option for arthroscopic
Bankart repair. ${ }^{[13]}$ There are few clinical studies in the literature related to all-suture anchors. Gül et al. ${ }^{[14]}$ reported the mean Rowe and Constant scores as $93.6 \pm 5.3$ and $92 \pm 4.3$, respectively, at the end of a mean 28.8 (range, 24 to 48 ) month follow-up period of 62 arthroscopic Bankart repairs performed with all-suture anchors, and stated the rate of redislocation to be $8.1 \%$. Lee et al. ${ }^{[7]}$ reported the mean Rowe scores as $87.9 \pm 14.9$ vs. $88.5 \pm 14.6$, respectively, at the end of a two-year follow-up period with the comparison of all-suture anchors and conventional biodegradable suture anchors in arthroscopic Bankart repair and no significant difference was determined between the two groups $(p=0.857)$. In the same study, the redislocation rates were observed to be similar at $6.1 \%$ and $5.9 \%$ for the all-suture anchor and conventional biodegradable suture anchor groups, respectively $(\mathrm{p}=0.682)$. In the present study, the mean Rowe scores of Group A and Group B were determined to be $89.2 \pm 13.8$ (range, 40 to 100) and $88.7 \pm 16.9$ (range, 25 to 100), respectively, and the mean Constant scores were $87.2 \pm 8.9$ (range, 48 to 96 ) and $86.9 \pm 9.0$ (range, 46 to 96 ), respectively. The redislocation rates in the current study were $3.1 \%$ in Group A and 2.9\% in Group B, and the apprehension test positivity rates were $6.3 \%$ and $8.6 \%$, respectively. In these respects, the outcomes of the present study appear to be consistent with the literature and the study hypothesis.

With rapid developments in the orthopedic materials industry, a wide range of anchor designs has been developed. As the range is wide, it is difficult for an orthopedic surgeon to decide which anchor should be used. Each type of anchor has specific advantages and disadvantages. Metal suture anchors have disadvantages such as migration, chondral damage, loosening, incarceration of the metal implant within the joint, and particularly difficulties in postoperative imaging methods such as MRI. ${ }^{[15-17]}$ In consideration of these complications, bioabsorbable suture anchors were developed. Although these anchors managed to prevent many of the complications associated with metal suture anchors, they entailed other complications such as reactive synovitis, loss of fixation due to early degradation, and osteolysis due to increased inflammation. ${ }^{[18]}$ It has been reported that although all-suture anchors, which have been used in recent years, have disadvantages such as tunnel expansion and cyst formation, satisfactory short-term clinical outcomes have been published and these disadvantages have been shown not to have affected the clinical outcomes. ${ }^{[5,7]}$ Although the long-term outcomes are still unknown, there are advantages implying that all-suture anchors may maintain glenoid bone stock, improve the quality of postoperative 
imaging methods, and facilitate revision surgery if necessary. ${ }^{[5,19]}$ In addition, all-suture anchors without rigid components enable correct and simple anchor placement without damaging the subscapularis. ${ }^{[4]}$

Martel et al. ${ }^{[20]}$ reported the development of glenohumeral osteoarthritis in four of 41 patients following a mean 33 (range, 12 to 47) month follow-up period after arthroscopic Bankart repair performed with metal suture anchors. Ikemoto et al. ${ }^{[21]}$ reported osteoarthritis development at the rate of $4 \%$ with bioabsorbable suture anchors and $21.5 \%$ with metal suture anchors after a follow-up period of mean 62 (range, 24 to 106) months after arthroscopic Bankart repair. No osteoarthritis development was observed in any patient in either of the two groups in the present study. Although the results of the current study differ from the literature in this respect, osteoarthritis development is associated with several variables such as age at first dislocation, age at surgery, the time from first dislocation to surgery, the total number of dislocations, and the number of anchors used. ${ }^{[2]}$

There were some limitations to this study. First, although the data of patients treated with all-suture anchors were collected prospectively, this was not a randomized controlled comparative study because the clinical use of the two types of anchors occurred in different periods. For this reason, seven patients treated with metal suture anchors were excluded from the study as the follow-up period exceeded five years, and 19 patients with follow-up periods of less than two years or who were lost to follow-up could not be evaluated in this retrospective study. However, strong aspects of the study can be considered to be that all the patients were operated on by the same surgeon with a similar technique and the two groups had similar demographic characteristics. A second limitation was that since the institutional review board did not allow computed tomography or MRI to be performed, radiographic evaluation could only be made with direct radiographs. Therefore, no information could be provided regarding the disadvantages of all-suture anchors such as tunnel enlargement or cyst formation. In addition, the follow-up periods of this study may be relatively short to be able to observe the development of glenohumeral osteoarthritis. Nevertheless, this study had a relatively longer follow-up period with regard to the clinical outcomes of all-suture anchors compared to the literature.

In conclusion, satisfactory outcomes were obtained with the use of all-suture anchors in arthroscopic Bankart repair for traumatic anterior shoulder instability. All-suture anchors and metal suture anchors have similar outcomes in the mid-term, and all-suture anchors are a reliable and effective option for arthroscopic Bankart repair.

\section{Declaration of conflicting interests}

The authors declared no conflicts of interest with respect to the authorship and/or publication of this article.

\section{Funding}

The authors received no financial support for the research and/or authorship of this article.

\section{REFERENCES}

1. Zhang AL, Montgomery SR, Ngo SS, Hame SL, Wang JC, Gamradt SC. Arthroscopic versus open shoulder stabilization: current practice patterns in the United States. Arthroscopy 2014;30:436-43.

2. Lenters TR, Franta AK, Wolf FM, Leopold SS, Matsen FA 3rd. Arthroscopic compared with open repairs for recurrent anterior shoulder instability. A systematic review and meta-analysis of the literature. J Bone Joint Surg [Am] 2007;89:244-54.

3. Hobby J, Griffin D, Dunbar M, Boileau P. Is arthroscopic surgery for stabilisation of chronic shoulder instability as effective as open surgery? A systematic review and meta-analysis of 62 studies including 3044 arthroscopic operations. J Bone Joint Surg [Br] 2007;89:1188-96.

4. Lacheta L, Dekker TJ, Anderson N, Goldenberg B, Millett PJ. Arthroscopic Knotless, Tensionable All-Suture Anchor Bankart Repair. Arthrosc Tech 2019;8:e647-e653.

5. Willemot L, Elfadalli R, Jaspars KC, Ahw MH, Peeters J, Jansen N, et al. Radiological and clinical outcome of arthroscopic labral repair with all-suture anchors. Acta Orthop Belg 2016;82:174-8.

6. Sarıkaya B, Bozkurt C, Gül O, Bekin Sarıkaya PZ, Sipahioğlu S, Altay MA. Comparison of the clinical results of isolated Bankart and SLAP 5 lesions after arthroscopic repair. Jt Dis Relat Surg 2020;31:223-9.

7. Lee JH, Park I, Hyun HS, Kim SW, Shin SJ. Comparison of clinical outcomes and computed tomography analysis for tunnel diameter after arthroscopic bankart repair with the all-suture anchor and the biodegradable suture anchor. Arthroscopy 2019;35:1351-8.

8. Di Giacomo G, Itoi E, Burkhart SS. Evolving concept of bipolar bone loss and the Hill-Sachs lesion: from "engaging/non-engaging" lesion to "on-track/off-track" lesion. Arthroscopy 2014;30:90-8.

9. Wolf JM, Cameron KL, Owens BD. Impact of joint laxity and hypermobility on the musculoskeletal system. J Am Acad Orthop Surg 2011;19:463-71.

10. Rowe CR, Zarins B, Ciullo JV. Recurrent anterior dislocation of the shoulder after surgical repair. Apparent causes of failure and treatment. J Bone Joint Surg [Am] 1984;66:159-68.

11. Constant CR, Murley AH. A clinical method of functional assessment of the shoulder. Clin Orthop Relat Res 1987;214:160-4.

12. Samilson RL, Prieto V. Dislocation arthropathy of the shoulder. J Bone Joint Surg [Am] 1983;65:456-60.

13. Atik OŞ. Is there something new and interesting in my article? Eklem Hastalik Cerrahisi 2019;30:69. 
14. Gül O, Okutan AE, Ayas MS. Arthroscopic glenoid labral lesion repair using all-suture anchor for traumatic anterior shoulder instability: short-term results. J Shoulder Elbow Surg 2019;28:1991-7.

15. Dhawan A, Ghodadra N, Karas V, Salata MJ, Cole BJ. Complications of bioabsorbable suture anchors in the shoulder. Am J Sports Med 2012;40:1424-30.

16. Bek D, Ege T, Erdem Y, Tunay S. Severe cartilage loss caused by metallic anchors in surgical treatment of a Bankart lesion: report of three cases. Eklem Hastalik Cerrahisi 2015;26:116-9.

17. Ozbaydar M, Elhassan B, Warner JJ. The use of anchors in shoulder surgery: a shift from metallic to bioabsorbable anchors. Arthroscopy 2007;23:1124-6.

18. Park MJ, Hsu JE, Harper C, Sennett BJ, Huffman GR. Poly$\mathrm{L} / \mathrm{D}$-lactic acid anchors are associated with reoperation and failure of SLAP repairs. Arthroscopy 2011;27:1335-40.
19. Agrawal V, Pietrzak WS. Triple labrum tears repaired with the JuggerKnot ${ }^{\mathrm{TM}}$ soft anchor: Technique and results. Int J Shoulder Surg 2015;9:81-9.

20. Martel ÉM, Rodrigues A, Dos Santos Neto FJ, Dahmer C, Ranzzi A, Dubiela RS. Evaluation of postoperative results from videoarthroscopic treatment for recurrent shoulder dislocation using metal anchors. Rev Bras Ortop 2016;51:45-52.

21. Ikemoto RY, Murachovsky J, Nascimento LGP, Bueno RS, Almeida LHO, Kojima C. Evaluation of surgical treatment of patients with shoulder instability. Acta Ortop Bras 2017;25:266-9.

22. Franceschi F, Papalia R, Del Buono A, Vasta S, Maffulli N, Denaro V. Glenohumeral osteoarthritis after arthroscopic Bankart repair for anterior instability. Am J Sports Med 2011;39:1653-9. 